\title{
CO-PLANAR CRACK INTERACTION IN CLEAVED MICA
}

\author{
J.C. Hill ${ }^{1}$, S.J. Bennison ${ }^{2}$, P.A. Klein ${ }^{3}$, A. Jagota ${ }^{2}$, S. Saigal ${ }^{1}$ \\ ${ }^{1}$ Department of Civil \& Environmental Engineering, Carnegie Mellon University, Pittsburgh, (USA) \\ ${ }^{2}$ CR\&D, Experimental Station, The DuPont Company, Wilmington Delaware 19880-0356 (USA) \\ ${ }^{3}$ Sandia National Laboratories, Livermore, California 94551 (USA)
}

\begin{abstract}
This paper describes experimental work on cleavage of mica in a double cantilever beam (DCB) geometry in which the main crack induced by a wedge driven into one side of the specimen interacts with a pre-existing internal crack. The latter is introduced by inserting a fiber between the delaminating beams of the same mica specimen during a previous DCB experiment, followed by retraction of the wedge, which results in healing of the beams except for an internal crack wedged apart by the inserted fiber. The experiment has been simulated numerically using a cohesive zone model, implemented as cohesive elements, to represent the separating interface. As the main crack approaches the internal preexisting crack, the two interact, resulting in mutual repulsion that manifests as an increase in apparent toughness. This feature of the experiment is captured very well by the model, independent of cohesive zone parameters other than the work of fracture. At a critical value of wedge displacement, the two cracks coalesce unstably, and the simulation captures this event as well. However, the instability condition depends on additional details of the cohesive zone law; by matching simulation to experiment one is able to extract a characteristic cohesive zone opening or peak cohesive stress.
\end{abstract}

KEYWORDS cohesive zone model, mica, instability, fracture.

\section{INTRODUCTION}

Cleavage experiments on mica by insertion of a wedge, a double cantilever beam (DCB) geometry, go back to the work of Obreimoff [1], have been re-visited often [2], and have contributed greatly to our understanding of the physics of fracture. Here we report on DCB experiments in mica in which a primary crack, induced by the wedge, interacts with a second, internal crack. These experiments build on the work of Wan et al. [3-5] on mica where, in particular, a repulsive interaction of co-planar cracks was observed. With increasing wedge insertion the two cracks coalesce eventually into one. The experiments have been simulated successfully using a cohesive zone model for the interfacial separation process. They reveal that the instability corresponding to crack coalescence depends on details of the cohesive zone model in addition to the fracture energy. By obtaining agreement between the experiments and simulation, one is able to extract a measure of the characteristic cohesive zone opening. 
The cohesive zone approach to fracture, introduced originally by Barenblatt \& Dugdale [6,7], has recently received considerable renewed attention $[8,9,10]$. It is especially powerful when implemented for numerical simulation of fracture, and is able to model different forms of inelasticity, crack nucleation and propagation. In particular, it has the potential to extend fracture analysis to small dimensions, and possibly to be a mechanism to bridge length scales. The cohesive zone approach requires the specification of a model that describes tractions resisting separation of material points at a crack tip. In an ideal elastic material, the macroscopic mechanics of a propagating crack when the cohesive zone is small compared to all dimensions are governed by the work of fracture alone [2]. Other cohesive zone parameters affect only the details of the stress and displacement fields near the crack tip. In inelastic materials, however, the work of fracture alone is insufficient; at least a second parameter is needed, such as peak separation stress or characteristic opening displacement. Even for a crack in an ideal elastic material, these details of the cohesive zone model can be important for crack initiation or certain instabilities.

\section{EXPERIMENTAL PROCEDURE AND RESULTS}

\section{Material Selection and Preparation}

Muscovite mica, an aluminum silicate, is a layered mineral with strong covalent intra-layer bonds and weak interlayer bonds. Because of this crystallographic structure, mica cleaves naturally. In addition, because muscovite mica is optically transparent, crack length measurements can be made by viewing interference fringes through the thickness. DCB specimens, $50 \mathrm{~mm} \times 10 \mathrm{~mm} \times 0.15 \mathrm{~mm}$ in dimension, were cut from single crystal muscovite mica sheets using a precision saw. To introduce an internal coplanar crack, the specimen was cleaved a distance of approximately $25 \mathrm{~mm}$. A $7 \mu \mathrm{m}$ carbon fiber was inserted across the width of the specimen and the cleaved mica was allowed to reheal. The fiber created a well-defined internal crack at the interface.

\section{Experimental Test Procedure}

Figure 1 illustrates the experimental apparatus. A crack was initiated in the prepared specimen using a $270 \mu \mathrm{m}$ thick blade along the plane of the internal crack. Specimens with significantly asymmetric beams after crack initiation or with cracks on different cleavage planes were discarded. The cleaved mica was placed in the specimen holder and the wedge was adjusted vertically to minimize asymmetric displacements. A stepper motor pushed the mica specimen onto the blade in one-micron increments at a velocity of 20 microns per second. The crack propagation was viewed with an inverted optical microscope using green light with a wavelength of $550 \mathrm{~nm}$. Crack lengths, measured from the fixed contact point of the wedge, were obtained using a micrometer attached to the microscope stage.

\section{Fracture Energy Measurements}

Based on beam theory, the relationship between the measured crack length $c$ and the mechanical energy release rate $G$ in a DCB configuration can be expressed as [11]

$$
G=\frac{3 E h^{3} d^{2}}{4 c^{4}}
$$

where $E$ is Young's modulus, $h=\left(\left(h_{1}^{3}+h_{2}^{3}\right) / 2\right)^{1 / 3}$ is the effective specimen thickness, and $2 d$ is the wedge thickness. The modulus of elasticity for muscovite mica, as reported in the literature, varies from as low as $54.9 \mathrm{GPa}$ [4] to as high as $196 \mathrm{GPa}$ [1]. In this work, the Young's modulus has been taken to be $169 \mathrm{GPa}$, as measured by McNeil and Gremsditch [12] using Brillouin scattering. 


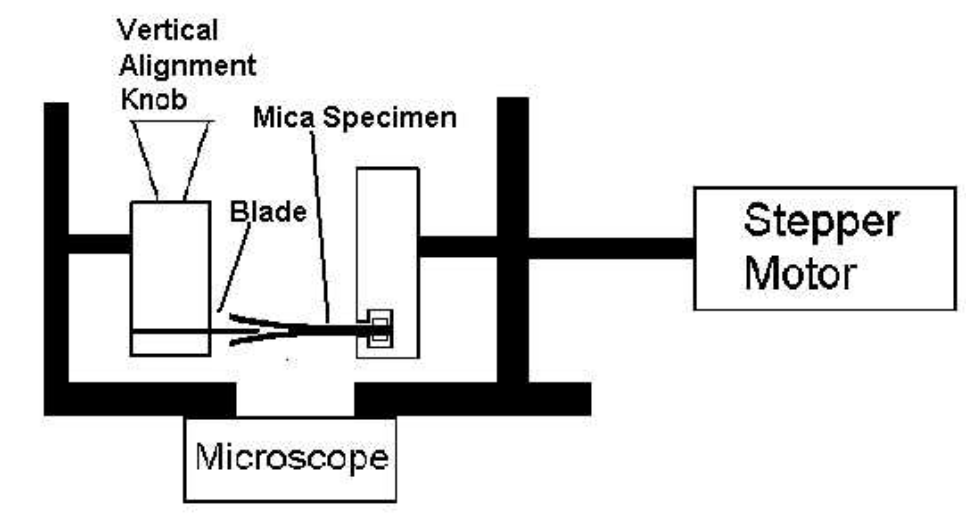

Figure 1: Constant displacement double-cantilever beam experimental apparatus. The vertical alignment knob is used to align the blade to create a symmetric crack. The stepper motor advances the mica specimen at $20 \mu \mathrm{m}$ per second on the blade ( $\sim 270 \mu \mathrm{m}$ thick). The ensuing crack propagation is viewed using an inverted optical microscope.

Define an apparent fracture energy, $G^{*}$, as

$$
G^{*}=\frac{3 E h^{3} d^{2}}{4 c_{1}{ }^{4}},
$$

based on the length of the primary crack, $c_{l}$, due to the inserted wedge. $G^{*}$ equals the fracture toughness $G_{c}$ when the two cracks do not interact. When they do, it's departure from $G_{c}$ measures the repulsion or attraction between the two cracks.

A typical crack interaction sequence is illustrated in Figure 2. Figure 2(a) shows the propagating DCB crack on the left approaching the crack front due to the inserted fiber, on the right. Note that in Figures 2(a) and 2(b), the distance between the two crack fronts remains roughly constant, though the crack front caused by the fiber has been repelled. In Figures 2(c-e), the distance between the two crack fronts continues to decrease until, just before Figure 2(f), the cracks coalesce.

Figure 3 shows the measured apparent fracture energy versus the wedge position. Initially, the apparent fracture energy $G^{*}$ is approximately $800 \mathrm{~mJ} \mathrm{~m}^{-2}$, its value for the healed interface. As the propagating crack front approaches the internal crack, the apparent fracture energy increases, then drops abruptly, and finally increases again to a value $\approx 1300 \mathrm{~mJ} \mathrm{~m}^{-2}$, the fracture energy of virgin, uncleaved muscovite mica.

\section{NUMERICAL SIMULATION}

The experiment has been simulated numerically using the commercial finite element code, ABAQUS ${ }^{\circledR}$ [13], augmented with cohesive elements that model the separating interface [10]. The beams were modeled using two-dimensional plane strain elements; the simulation has been conducted as an implicit dynamic procedure to allow one to capture the instability. The dimensions and material properties reproduce the experimental specimen, i.e. length, $\mathrm{L}=20 \mathrm{~mm}$, beam thickness, $\mathrm{h}_{1}=133 \mu \mathrm{m}$, $\mathrm{h}_{2},=84 \mu \mathrm{m}$, wedge half-thickness, $\mathrm{d}=133.5 \mu \mathrm{m}, \mathrm{E}=169 \mathrm{GPa}, v=0.3, \mathrm{G}_{1}=820 \mathrm{~mJ} / \mathrm{m}_{2}, \mathrm{G}_{2}=1250$ $\mathrm{mJ} / \mathrm{m} 2$. 
(a)
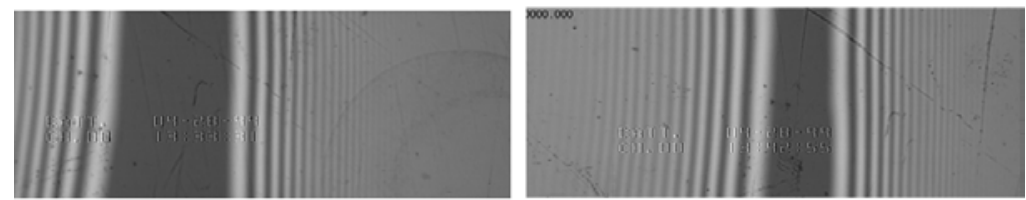

(d)

(b)
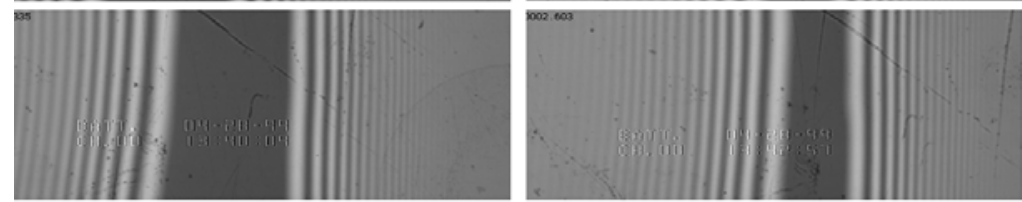

(e)
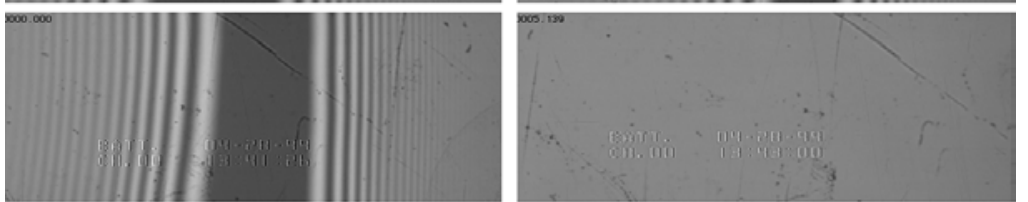

$(f)$

Figure 2: Series of experimental pictures illustrating crack repulsion. Crack front due to inserted wedge is on the left and crack front due to inserted fiber is on the right. Wedge positions relative to the fiber are (a) $-9.51 \mathrm{~mm}$, (b) $-9.23 \mathrm{~mm}$, (c) $-8.98 \mathrm{~mm}$, (d) $-8.69 \mathrm{~mm}$, (e) $-8.64 \mathrm{~mm}$, (f) $-8.59 \mathrm{~mm}$

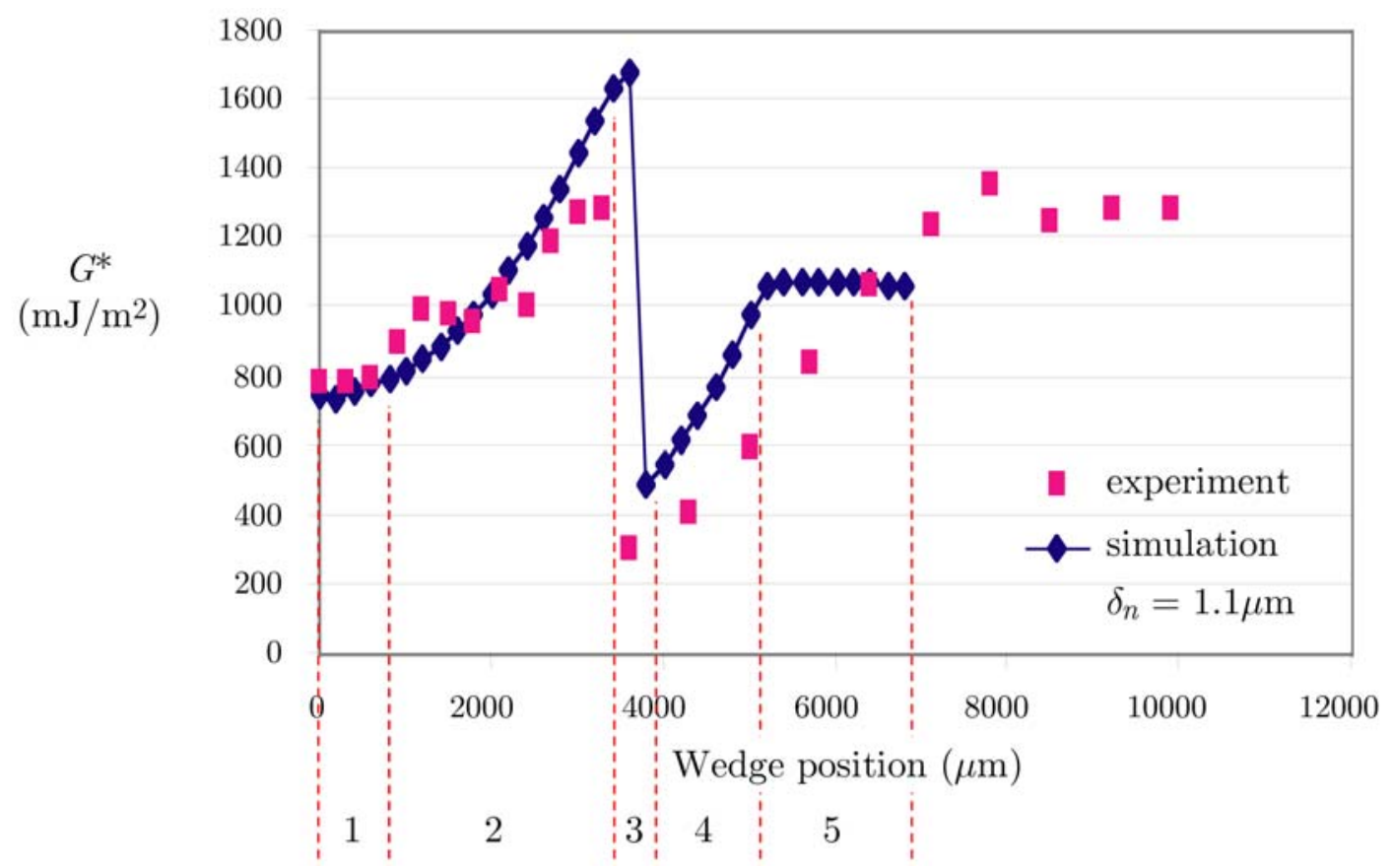

Figure 3: Measured and simulated apparent fracture toughness as a function of wedge position. Coalescence of the two cracks is simultaneous with the significant drop in apparent fracture energy. In the simulation, $\delta_{\mathrm{cr}}=1.1 \mu \mathrm{m}$.

The fracture zone has been modeled using a simplified version of the phenomenological cohesive law proposed by $\mathrm{Xu}$ and Needleman [8]. Tractions, $T_{n}$ and $T_{t}$, resisting relative displacements in opening and shear are calculated from a potential function $\Phi$, given as

$$
\Phi\left(\Delta_{n}, \Delta_{t}\right)=\phi\left\{1-\exp \left(-\frac{\Delta_{n}}{\delta_{c r}}\right)\left[1+\frac{\Delta_{n}}{\delta_{c r}}\right] \exp \left(-\frac{\Delta_{t}^{2}}{\delta_{c r}^{2}}\right)\right\}
$$




$$
T_{n}=-\frac{\partial \phi}{\partial \Delta_{n}} ; T_{t}=-\frac{\partial \phi}{\partial \Delta_{t}},
$$

where $\delta_{\mathrm{cr}}$ is a characteristic opening of the cohesive zone, $\Delta_{\mathrm{n}}$ and $\Delta_{\mathrm{t}}$ are the normal and tangential relative displacements, and $\phi$ is fracture toughness. The maximum cohesive stress, $\sigma_{\max }$, fracture toughness, and $\delta_{\mathrm{cr}}$ are related through

$$
\sigma_{\max }=\phi /\left(\delta_{c r} \exp (1)\right) \text {. }
$$

The geometry used for the simulations was taken from measured specimen dimensions. A value of $\phi=$ $820 \mathrm{~mJ} / \mathrm{m}^{2}$ was used for the cohesive elements. This represents the measured fracture energy of the healed mica-mica interface, and is lower than that of uncracked mica. The carbon fiber inclusion was modeled by a circular rigid surface in contact with the continuum elements representing the beams. Wedge displacement was imposed by applying displacement boundary conditions to successive sets of nodes on the beams at the appropriate location, remote from the crack tip.

\section{DISCUSSION \& CONCLUSIONS}

A comparison of simulated and experimental results is shown in Figure 3, for a critical cohesive zone opening, $\delta_{\mathrm{cr}}=1.1 \mu \mathrm{m}$. Also indicated in the figure are five notional stages. In stage 1, the interaction of the two cracks is minimal. In stage 5 there is only one crack in the system. In both, the apparent fracture toughness does not change with wedge location. Its value in stage 1 is estimated well by the simulation; this confirms that the simulation procedure is accurate enough to capture a simple DCB experiment. The under-prediction of fracture toughness in stage 5 is likely related to our use of a much coarser mesh in that region since the primary interest has been to capture the response prior to the instability, and the instability itself. Stage 2 represents the mutual repulsion between the cracks, which manifests as an increase in apparent toughness that is captured well by the simulation, although the simulated value exceeds the measured value. The simulation captures stage 3 , the instability that leads to coalescence of the two cracks. After coalescence, the recovery to the uncleaved fracture energy is gradual, not immediate. We find, in the simulations, that the crack retains contact with the cylindrical fiber after coalescence, which results in a longer crack length than would be predicted due to the wedge alone, and hence a lower apparent fracture toughness. One may surmise that the same occurs in the experiment.

The numerical simulation captures all the features of the experiment very well qualitatively, with reasonably good quantitative agreement, generally. However, the value of critical opening parameter, $\delta_{\mathrm{cr}}$, required to do so, $1.1 \mu \mathrm{m}$, is much larger than might be expected for a brittle material. Figure 4 shows the effect of $\delta_{\mathrm{cr}}$ on simulation results. Larger values result in earlier onset of the coalescence instability. It is interesting to note that whereas all other features of this experiment appear to be controlled solely by the energy release rate, the instability itself depends on additional parameters of the cohesive zone model. Two questions remain unanswered. Why is the value of $\delta_{\text {cr }}$ required to attain agreement between experiment and simulation so large? How do the conditions for coalescence instability scale with cohesive zone parameters? We have not resolved either satisfactorily at this time, but consider either capillary condensation or interaction between large charged domains as possible explanations for the former. Progress towards answering the latter question can likely be made by approximate analyses of the experiment using beam theory. 


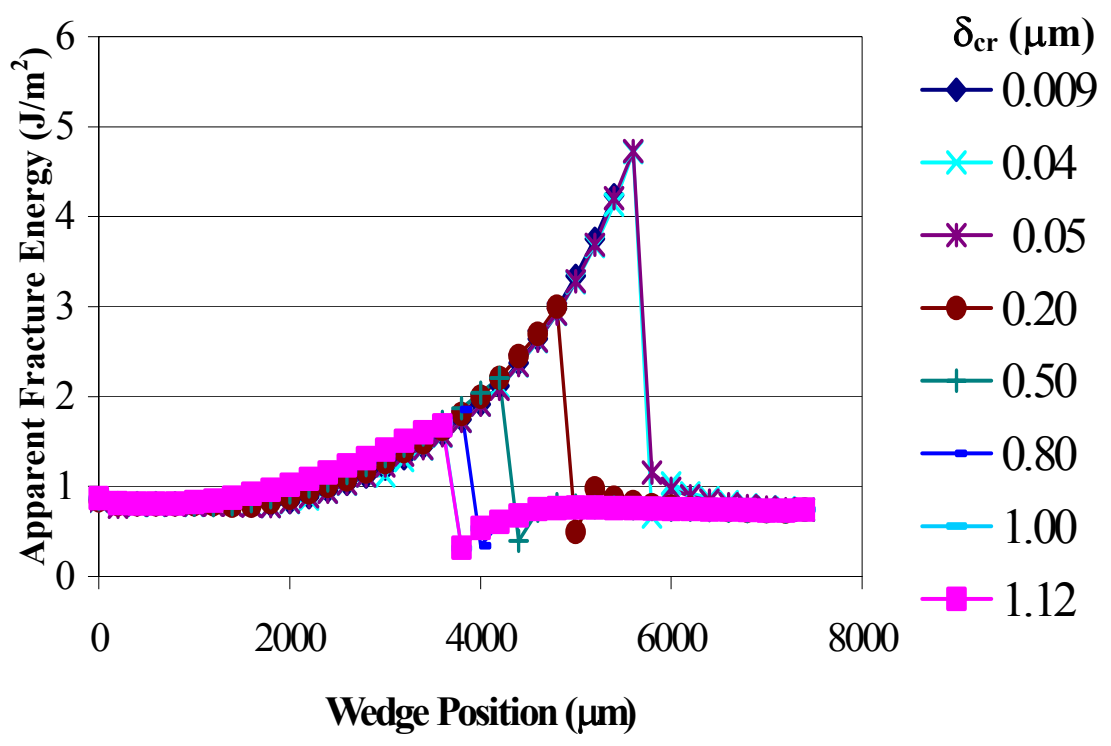

Figure 4: Effect of critical opening, $\delta_{\text {cr }}$ on the instability leading to coalescence of the two cracks.

\section{ACKNOWLEDGEMENTS}

This work was supported in part by the Computational Sciences Graduate Fellowship Program. Part of the work was carried out under the auspices of the Summer Institute of Sandia and under the NSFSandia Life Cycle Engineering Initiative. We'd also like to acknowledge the help with the experimental work of J.B. Brown \& R.G. Bender at DuPont CR\&D.

\section{REFERENCES}

1. Obreimoff, J.W. (1930) Proc. Roy. Soc. Lond. A217: 905.

2. Lawn, B. (1993), Fracture of Brittle Solids, Cambridge University Press.

3. Wan, K.T., Lathabai, S., \& Lawn, B.R. (1990) J. Eur. Cer. Soc. 6(4): 259-268

4. Wan, K.T. and B.R. Lawn. (1992) Acta. Metall. 40(12).

5. Wan, K.T., Lawn, B.R., \& Horn, R.G. (1992) J. Mat. Res. 7(6): 1584-1588.

6. Barenblatt, G.I. (1959) Appl. Math. Mech. 23: 622-636.

7. Dugdale, D.S. (1960) J. Mech. Phys. Solids 8: 100-104.

8. Xu, X.-P. and Needleman, A. (1994) J. Mech. Phys. Solids 42: 1397-1434.

9. Camacho, G.T. and Ortiz, M. (1996) Int J Solids Structures 33:20-22: 2899-2938.

10. Rahulkumar, P., Jagota, A., Bennison, S.J., and Saigal, S. Int. J. Solids Struct., 37 [13] 18731897 (2000).

11. Rice, J.R., (1968) in Fracture, Liebowitz H. (editor), Academic Press, 191-311.

12. McNeil, L.E., Grimsditch, M., (1993) J. Phys.: Cond. Mat. 5(11): 1681-1690.

13. ABAQUS ${ }^{\circledR}$, v 5.8, Hibbit, Karlsson \& Sorensen, Inc., Pawtucket, Rhode Island (USA). 